\title{
METAL-POLYMER-METAL SYSTEM IN THE HIGH STATIC ELECTRIC FIELD
}

\author{
M. RADNY \\ Institute of Experimental Physics, University of Wroclaw \\ Cybulskiego 36, 50-205 Wrocław, Poland \\ AND A. KaRPowICZ
}

Institute of Electrical Engineering and Technology, Wrocław Technical University

pl. Grunwaldzki 13, 50-377 Wrocław, Poland

(Received June 14, 1991; in revised form September 9, 1991)

The negative differential resistance phenomenon evident on current-voltage $(I-V)$ characteristics for Al-plasma-polymerized polystyrene-Al system is described by means of the Schottky-like barrier model constructed directly from the simplified electronic band structure of an insulator.

PACS numbers: $73.40 . \mathrm{Rw}, 73.40 . \mathrm{Gk}$

\section{Introduction}

Probably the first structure in which the negative differential resistance (NDR) effect has been observed was a tunnel diode [1]. The similar characteristic has been observed later in a metal-oxide-semiconductor tunnel junction [2] and then in the most artificial structures - multilayers [3].

The negative resistance phenomenon in multilayer structures can be easily understood in terms of the resonant tunneling [4]. In the case of a double barrier heterostructure (Fig. 1a) an electron tunneling transmission coefficient is enhanced when the energy of the tunneling electrons coincide with the bound state energy level $\left(E_{\mathrm{b}}\right)$ within the well (region II). While increasing the bias voltage, the bound state energy level drops below the conduction band edge of region I. The electron must tunnel through the entire barrier structure to get the free states of region III in Fig. 1a giving rise to the drop in the current, so that $I-V$ characteristic would show the negative resistance.

The origin of the NDR effect in the tunneling diode and metal-oxide-semiconductor junction seems to be quite different. According to Zener's idea of the interband tunneling [5] the tunneling process in these junctions can occur between a filled band on the metal side to a next higher energy band on the semiconductor side by the application of the electric field (metal-oxide-semiconductor junction 

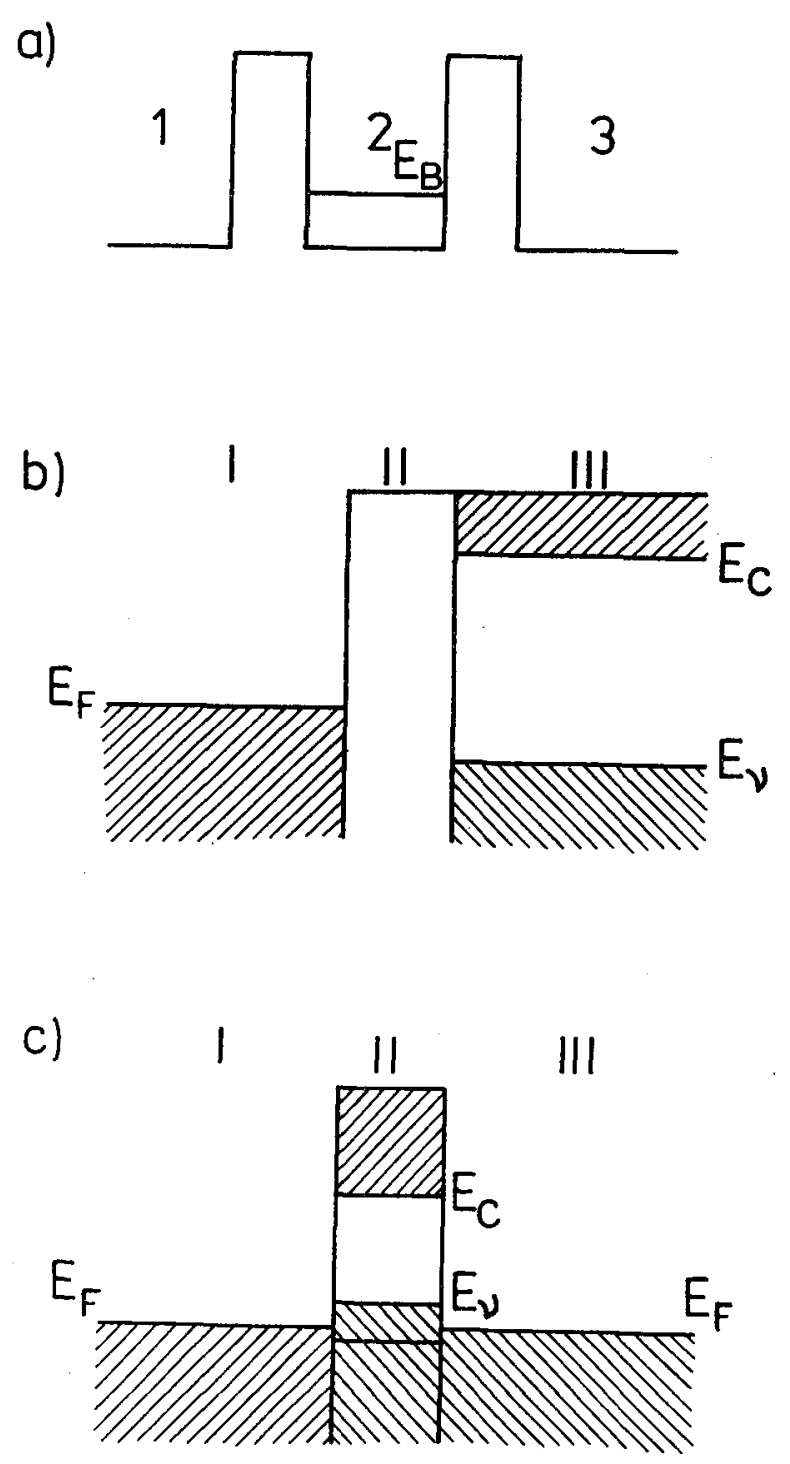

Fig. 1. Energy diagrams at zero bias voltage for the (a) double barrier heterostructure; $E_{\mathrm{b}}$ denotes a bound state within the well, (b) metal(I)-oxide(II)-semiconductor(III) junction, (c) metal-polymer-metal junction; metal (I,III) and insulator (II) are represented by one- and two-band model, respectively. $E_{\mathrm{F}}$ denotes the Fermi level in metal while $E_{\mathrm{c}}$ and $E_{\mathrm{v}}$ are the boundary of the insulator (semiconductor) energy gap .

- Fig. 1b). The negative resistance occurs due to the semiconductor energy band gap which is treated as a potential barrier. In the Schottky model which assumes that there are no localized states (surface, impurity, etc.) that can modify the 
interface formation the height of this barrier is equal to the difference between the bottom of the semiconductor conduction band and the Fermi energy in the metal.

The system which we want to discuss here consists of two metallic media (aluminum) separated by an insulator (plasma-polimerized polystyrene - Section 2) with the wide energy gap in the appropriate electron band structure. In Fig. 1c the simplest electronic band structure of the system, i.e. one-band and two-band model for the metal and insulator, respectively, is presented. It is easy to see that although negative differential resistance has been observed in this system (Section 2) no one of the described above pictures is applicable in this case. In Section 3 we show that on the base of the classical Schottky model and taking into account the complex dispersion relation in the insulator energy gap it is possible to construct a Schottky-like potential barrier model appropriate for the system investigated here.

\section{Experimental results}

The metal-insulator-metal structure (MIM capacitor) was prepared in the following way. One of the aluminum electrode was vacuum evaporated onto a glass substrate through the mask. Next, the insulator layer was deposited onto the substrate by polymerization of styrene in glow discharge. In order to make the second electrode, the layer of plasma-polymerized polystyrene (p-p PS) was masked and then aluminum electrode was evaporated in the vacuum. Finally, such prepared structure was connected with the measuring apparatus by using a silver-coated springs [6].

At first, current-time dependent characteristics (current absorption characteristics) were taken for increasing value of the applied electric field as a fixed parameter. The applied field was in the range of $10^{3} \mathrm{~V} / \mathrm{m}$ up to the breakdown value of the sample. Next, the current-electric field characteristics were determined for a constant value of time of the current absorption characteristics. The character of the obtained current-electric field line does not depend on the chosen value of time in the steady-state range of the current absorption characteristics. Dielectric parameters of the films were as follows:

$$
\begin{array}{ll}
320 \mathrm{~nm} \quad \varepsilon=2.4 & \tan \delta=1.6 \times 10^{-3}, \\
860 \mathrm{~nm} \quad \varepsilon=3.1 & \tan \delta=7.6 \times 10^{-3},
\end{array}
$$

where $\varepsilon$ is the relative dielectric constant.

It is shown in Fig. 2 that the conductivity of the polymer is a strong function of the electric field in the applied field range. Three specific regions have been distinguished for which the current-voltage characterstics can be connected with different conduction mechanisms. For electric field $\left(<4 \times 10^{7} \mathrm{~V} / \mathrm{m}\right)$ the current is a linear function of the field. In the field range of $4 \times 10^{7}$ to $8 \times 10^{7} \mathrm{~V} / \mathrm{m}$ the differential conductivity of the film is negative. For the strong electric fields $\left(>8 \times 10^{7} \mathrm{~V} / \mathrm{m}\right)$ the current is again the linear function of the applied electric field [6]. 


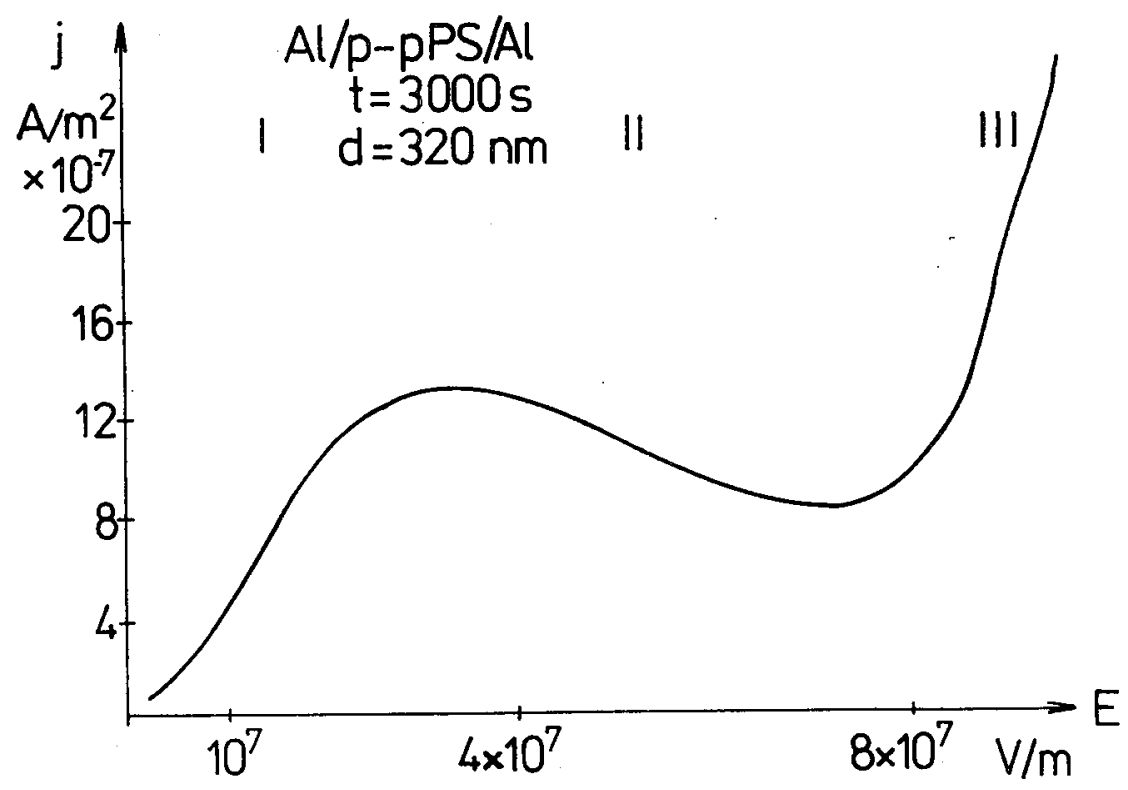

Fig. 2. Current-voltage characteristics for $\mathrm{Al} / \mathrm{p}-\mathrm{p} \mathrm{PS} / \mathrm{Al}$ system (see text).

\section{Potential barrier model of metal-insulator junction}

In order to describe the metal-insulator-vacuum system we adopt a one-band free-electron model for the metal and a two-band model for the insulator.

The metal is represented by a semi-infinite free electron model (Fig. 1c) with a finite surface barrier located at $x=0$ whose height is determined by the metal work function $\varphi$ plus the Fermi energy $E_{\mathrm{F}}$.

The insulator is represented by a simple two-band model where an explicit expression for the complex dispersion relation was used. Namely, the wave function

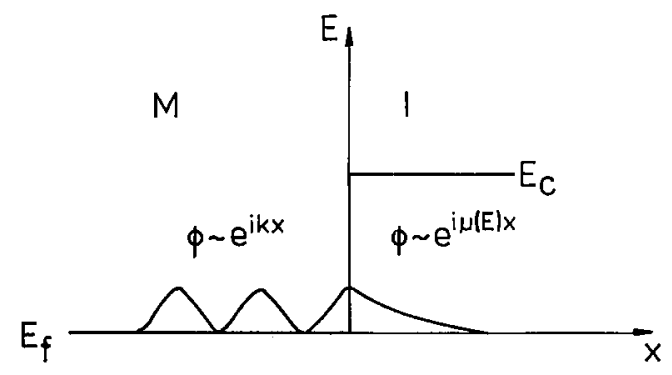

Fig. 3. Barrier heiglt for electrons incident from metal (M) of an energy $k^{2}=2 E_{F}$ at the insulator (I) band gap depends on the dispersion relation in the insulator band gap $[\mu(E)]$ (see text). 
of the electron incident at the interface from the metal $(x<0)$ with the energy at the forbidden energy gap of the insulator acquires an exponentially attended tail into barrier (Fig. 3)

$$
\psi \sim \exp [-\mu(E) x], \quad x>0
$$

where $\mu(E)$ is the imaginary component of the wave vector

$$
k_{x}=\pi / a+\mu(E) \text {. }
$$

As can be seen from Fig. $4, \mu(E)$ varies with the energy over the gap. The

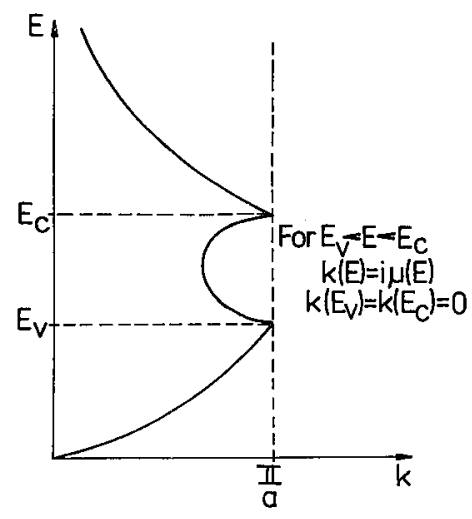

Fig. 4. Dispersion relation $E(k)$ in the simple two-band model of the insulator. Complex part of $E(k)$ in the band gap is characterized by $\mu(E)$ - the imaginary part of the wave vector.

explicit energy dependence of this quantity can be easily obtained from the simple two-band nearly free-electron model $[7,8]$

$$
\mu(E)=\sqrt{2 E+2(\pi / \alpha)^{2}-\sqrt{(E \pi / \alpha)^{2}+4 V_{1}^{2}}}
$$

where $E$ is an energy of electron, $V_{1}$ is a half value of energy gap of an insulator, and $\alpha$ is the lattice constant.

The metal-insulator interface can be represented now by the following model: The interface is assumed to be a plane across which the metal potential and dielectric constant change abruptly. As was mentioned earlier, the energy gap in the electronic structure of the insulator can be treated as the potential barrier at the interface. In order to determine its height we use the expression for the imaginary component of the wave vector $\mu(E)$ assuming its identity with the solution of the wave equation inside the finite potential barrier of arbitrary height $V$

$$
\psi \approx \exp \left(-k_{0} x\right)
$$

where $k_{0}=[2 m(V-E)]^{1 / 2}$. Ilence, from Eq. (1)

$$
\psi \approx \exp \left\{-\left[2 m^{*}(V-E)\right]^{1 / 2} x\right\} \approx \exp [-\mu(E) x]
$$

and

$$
\exp [-\mu(E) x]=\exp \left(-k_{0} x\right) .
$$




$$
\begin{aligned}
& \text { Finally, } \\
& k_{0}=\mu(E) \quad \text { or } \quad 2 m^{*}(E-V)=\mu^{2}(E),
\end{aligned}
$$

where $m^{*}$ is an effective mass of an electron. From the above equation the high of the Schottky barrier $(V)$ is energy dependent and because

$$
V\left(E_{\mathrm{c}}\right)=V\left(E_{\mathrm{v}}\right)=0
$$

one can easily obtain (see Fig. 5)

$$
V(E)= \begin{cases}\mu^{2}(E) / 2 m^{*}+E-E_{\mathrm{v}} & \text { for } \quad E_{\mathrm{v}} \leq E \leq E_{\max } \\ \mu^{2}(E) / 2 m^{*}+E_{\mathrm{c}}-E & \text { for } \quad E_{\max } \leq E \leq E_{\mathrm{c}}\end{cases}
$$

where $E_{\max }$ is an energy value for which $\mu(E)$ has maximum value.
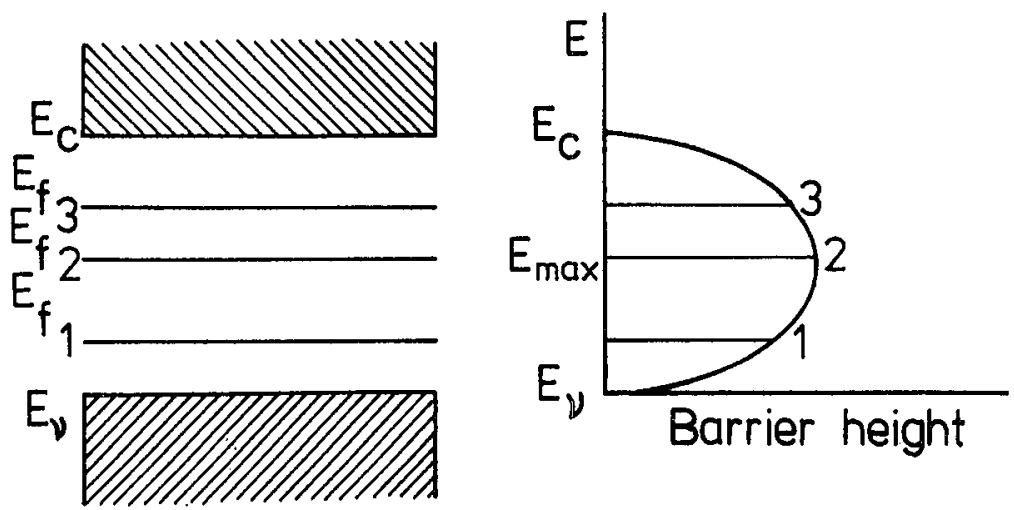

Fig. 5. Dependence of the barrier height at the metal-insulator junction on the metal Fermi level position in the energy gap of the insulator.

Let us notice the properties of this model. If $E=E_{\mathrm{F}}$ for $E>E_{\max }$ the above expressions are very close to the previous one defined simply as a difference between the Fermi level of the metal and bottom of the insulator conduction band. On the other hand, when the electron energy is $E<E_{\max }$ the height of the barrier decreases because $V\left(E_{\mathrm{v}}\right)=0$.

\section{Discussion}

The height of the metal-insulator barrier defined above explains well an origin of the negative differential resistivity of $\mathrm{Al} / \mathrm{p}-\mathrm{p} \mathrm{PS/Al} \mathrm{system} \mathrm{(Fig.} \mathrm{2).}$

For two metallic electrodes which are separated by an insulating film, the equilibrium conditions require the bottom of the conduction band of the insulator to be positioned above the Fermi level of the metal (but below the vacuum level - Fig. 1c). The height of the potential barrier is small and the current is a linear function of the bias voltage - region I in Fig. 2.

When the bias voltage is further increased and the Fermi level (left) coincides within the energy band gap of the insulator the barrier height increases. This is 
due to the fact that energy $E<E_{\max }$ and the conduction band edge is shifted down while increasing the bias voltage, so that the height of the potential barrier $V$ (Fig. 5 point 1 ) increases and then the current decreases. Hence, the tunneling probability, determined as $\exp \left[-2 d(V-E)^{1 / 2}\right]$ where $V$ and $d$ are the barrier height and width, respectively, decreases resulting in a decrease in the tunneling current. So the negative resistance is exhibited in the $I-V$ characteristic presented in Fig. 2 (region II). While increasing the voltage further, the Fermi level is still shifted up (relevant to the conduction band edge) where the electron energy is in the range $E_{\max }<E<E_{\mathrm{c}}$ (Fig. 5 points 2,3 ). In this case potential barrier height decreases, so that the current increases (Fig. 2 region III).

\section{Concluding remarks}

All different models proposed to explain the barrier formation of different junctions are, in a sense, a generalisation of two classical Schottky and Bardeen approximations [9]. In the discussion presented in this paper, media (aluminum, polymer) are assumed practically decoupled (classical Schottky model). However, the construction of the interface potential barrier to explain the negative differential resistance proposed here differs significantly from the models used before and to our knowledge has never appeared in the literature before. Moreover, the proposed mechanism is not restricted to the case discussed in this paper (metal-polymer-metal junction). It can also be easily used to other systems with the well-defined electronic band structure.

\section{References}

[1] L. Esaki, Phys. Rev. 109, 603 (1958).

[2] L.L. Chang, P.J. Stiles, L. Esaki, J. Appl. Phys. 38, 4440 (1967).

[3] L.L. Chang, L. Esaki, R. Tsu, Appl. Phys. Lett. 24, 593 (1974).

[4] D.K. Roy, Quantum Mechanical Tunneling and its Applications, World Scientific Publishing, Singapore 1986.

[5] A. Zener, Proc. R. Soc. Lond. A 145, 523 (1934).

[6] A. Karpowicz, S. Głuchowski, in Proc. 9th Symp. Electrical Discharges in Gases, Pasym (Poland) 1987, p.26.

[7] F. Forstman, Z. Phys. 235, 69 (1970).

[8] J.E. Inglesfield, B.W. Holland, in:The Chemical Physics of Solid Surfaces and Heterogeneous Catalysis, Eds. D.A. King, D.P. Woodruff, Elsevier Science Publishers, New York 1981.

[9] F. Flores, C. Tejedor, J. Phys. C 20, 145 (1987). 